\title{
Folgerecht nach dem Tod des Künstlers (Salvador Dalí) - Die Richtlinie 2001/84/EG und nationales Recht
}

EuGH, Urteil vom 15. April 2010 - C-518/08

\section{Vorabentscheidungsersuchen des Tribunal de Grande Instance de Paris (Frankreich), eingereicht am 27. November 2008 (Rechtssache C-518/08) (2009/C 32/34)}

\section{Vorlegendes Gericht}

Tribunal de Grande Instance de Paris

\section{Parteien des Ausgangsverfahrens}

Kläger: Fundació Gala-Salvador Dalí, Visual Entidad de Gestión de Artistas Plásticos

Beklagte: Société des Auteurs dans les Arts Graphiques et Plastiques, Juan-Leonardo Bonet Domenech, Eulalia-María Bas Dalí, María Del Carmen Domenech Biosca, Antonio Domenech Biosca, Ana-María Busquets Bonet, Mónica Busquets Bonet

\section{Vorlagefragen}

1. Kann Frankreich nach Erlass der Richtlinie vom 27. September 2001 (Richtlinie 2001/84/EG des Europäischen Parlaments und des Rates vom 27. September 2001 über das Folgerecht des Urhebers des Originals eines Kunstwerks 1 (ABI. L 272, S. 32) ein den Erben unter Ausschluss von Vermächtnisnehmern oder Rechtsnachfolgern vorbehaltenes Folgerecht beibehalten?

2. Gestatten die Übergangsbestimmungen des Art. 8 Abs. 2 und 3 der Richtlinie vom 27. September 2001 Frankreich eine Ausnahmeregelung?

\section{Schlussanträge der Generalanwältin Eleanor Sharpston vom 17. Dezember 2009 $^{1}$}

- 1. Im Jahr 1859 vollendete und verkaufte Jean-François Millet sein berühmtes Gemälde L'Angélus. Jahre nach seinem Tod, zu einer Zeit, als sich seine Familie, wie viele andere vom Ersten Weltkrieg Betroffene, in sehr schwierigen wirtschaftlichen Verhältnissen befand, wechselte das Gemälde seinen Besitzer zu einem Preis, der dem Verkäufer einen erheblichen Gewinn verschaffte. Es heißt, die Diskrepanz zwischen den beiden Sachverhalten habe den französischen Gesetzgeber im Jahr 1920 veranlasst, ein droit de suite oder Folgerecht einzuführen, wonach die Weiterveräußerung eines Kunstwerks dem Urheber oder seinen Erben Anspruch auf eine Vergütung verleiht. ${ }^{2}$

1 Originalsprache: Englisch. Im Internet abrufbar unter: http:// curia.europa.eu/.

2 Vgl. die Erklärung des Ministers für Kultur und Kommunikation am 16.3.2006 in der französischen Nationalversammlung (http:// www.assemblee-nationale.fr/12/cri/2005-2006/20060175.asp).
2. Seitdem hat das Recht auch in andere Rechtsordnungen Eingang gefunden. Es wurde 1948 auf fakultativer Grundlage in die Berner Übereinkunft ${ }^{3}$ aufgenommen und durch die Richtlinie 2001/84 (im Folgenden: Richtlinie) ${ }^{4}$ in der Europäischen Union verbindlich vorgeschrieben. Obwohl der Grundsatz einheitlich ist und die geltenden Sätze harmonisiert sind, verfügen die Mitgliedstaaten in verschiedener Hinsicht über einen Beurteilungsspielraum.

$3 \quad$ Berner Übereinkunft zum Schutz von Werken der Literatur und Kunst vom 9.9.1886, geändert vor allem in Brüssel am 26.6.1948. Alle Mitgliedstaaten der Europäischen Union sind Vertragsparteien der Übereinkunft.

4 Richtlinie 2001/84/EG des Europäischen Parlaments und des Rates vom 27.9.2001 über das Folgerecht des Urhebers des Originals eines Kunstwerks (ABl. L 272, S. 32). Obwohl der Begriff des droit de suite im Englischen weit verbreitet ist und insbesondere in der englischen Fassung der Berner Übereinkunft verwendet wird, werde ich im Folgenden, wie in der Richtlinie, den Begriff des Folgerechts verwenden. 
3. Nach gegenwärtigem Stand des französischen Rechts ist das Folgerecht nach dem Tod des Urhebers des Werks den gesetzlichen Erben des Urhebers unter Ausschluss aller testamentarischen Erben vorbehalten.

4. Der Künstler Salvador Dalí ${ }^{5}$ starb 1989, wobei er alle seine Rechte des geistigen Eigentums dem spanischen Staat vermachte. Wäre er ohne Hinterlassung eines Testaments gestorben, wären diese Rechte auf mehrere seitenverwandte Erben übergegangen.

5. Gemäß französischem Recht wurden die Folgerechtsvergütungen an den Verkäufen der Werke Dalís in Frankreich zugunsten jener seitenverwandten Erben erhoben. Zwischen der spanischen Gesellschaft, die Folgerechtsvergütungen für den spanischen Staat einzieht, vertreten durch eine von Dalí zu Lebzeiten gegründete Stiftung, und der französischen Gesellschaft zur Einziehung der Vergütungen, die Folgerechtsvergütungen an seine seitenverwandten Erben gezahlt hatte, entstand ein Rechtsstreit.

6. In diesem Zusammenhang fragt das Tribunal de grande instance de Paris, ob die französische Beschränkung des Kreises der Inhaber des Folgerechts auf gesetzliche Erben mit dem Unionsrecht vereinbar ist.

\section{Rechtlicher Rahmen}

\section{Die Richtlinie}

7. Im ersten Erwägungsgrund der Richtlinie wird festgestellt, dass das Folgerecht ein unabtretbares und unveräußerliches Recht ist, und im dritten Erwägungsgrund wird ausgeführt, dass es "den Urhebern von Werken der bildenden Künste eine wirtschaftliche Beteiligung am Erfolg ihrer Werke garantieren" soll und "[a]uf diese Weise ... ein Ausgleich zwischen der wirtschaftlichen Situation der bildenden Künstler und der Situation der anderen Kunstschaffenden hergestellt werden [soll], die aus der fortgesetzten Verwertung ihrer Werke Einnahmen erzielen“.

8. Der neunte Erwägungsgrund weist auf die frühere Rechtslage hin, nach der in den meisten Mitgliedstaaten ein Folgerecht vorgesehen war, das jedoch erhebliche Unterschiede, insbesondere in Bezug auf die erfassten Werke, die Anspruchsberechtigten, die Höhe des Satzes, die diesem Recht unterliegenden Transaktionen und die Berechnungsgrundlage aufweist. Weiter heißt es: „Die Anwendung oder Nichtanwendung eines solchen Rechts hat insofern erhebliche Auswirkungen auf die Wettbewerbsbedingungen im Binnenmarkt, als das Bestehen oder Nichtbestehen einer aus dem Folgerecht resultierenden Abführungspflicht ein Aspekt ist, der von jeder an dem Verkauf

$5 \quad$ Erwähnenswert ist, dass Dalí selbst vom L'Angélus stark beeinflusst war und 1963 unter dem Titel Le mythe tragique de l'Angélus de Millet eine ausführliche „paranoisch-kritische“ Auslegung des Werks veröffentlichte. eines Kunstwerks interessierten Person in Betracht zu ziehen ist. Daher ist dieses Recht einer der Faktoren, die zu Wettbewerbsverzerrungen und Handelsverlagerungen in der Gemeinschaft beitragen.

9. Ebenso wird im zehnten Erwägungsgrund hervorgehoben, dass „[d]iese Unterschiede hinsichtlich des Bestehens des Folgerechts und seiner Anwendung durch die Mitgliedstaaten ... unmittelbare negative Auswirkungen auf das reibungslose Funktionieren des Binnenmarkts im Sinne von Artikel 14 des Vertrags [haben], soweit er Kunstwerke betrifft", während nach dem elften Erwägungsgrund die Harmonisierung der Bestimmungen der Mitgliedstaaten zum Folgerecht zur Verwirklichung der unveräußerlichen Freiheiten des Binnenmarkts beiträgt. Folglich sollten nach dem 13. Erwägungsgrund „[b]estehende rechtliche Unterschiede ... beseitigt werden, soweit sie den Binnenmarkt in seiner Funktion beeinträchtigen, und es sollte verhindert werden, dass neue Unterschiede auftreten", ein Ziel, das vor allem im 14. und 15. Erwägungsgrund wiederholt wird. Zur Gewährleistung der Leistungsfähigkeit des Binnenmarkts im Bereich moderner und zeitgenössischer Kunstwerke müssen nach dem 23. Erwägungsgrund insbesondere die Sätze so weit wie möglich vereinheitlicht werden.

10. Allerdings heißt es im 13. Erwägungsgrund auch, dass „Unterschiede ..., die sich voraussichtlich nicht nachteilig auf den Binnenmarkt auswirken, ... bestehen bleiben [können] bzw. ... nicht verhindert werden [müssen]“, und im 15. Erwägungsgrund, dass „[e]ine Harmonisierung sämtlicher Bestimmungen der Mitgliedstaaten zum Folgerecht ... nicht erforderlich [erscheint]; damit so viel Spielraum wie möglich für einzelstaatliche Entscheidungen bleibt, genügt es [danach], nur diejenigen einzelstaatlichen Vorschriften zu harmonisieren, die sich am unmittelbarsten auf das Funktionieren des Binnenmarkts auswirken".

11. Desgleichen, allerdings genauer, wird im 27. Erwägungsgrund festgestellt: „Es erscheint angezeigt, unter Wahrung des Subsidiaritätsprinzips den Kreis der Personen zu bestimmen, denen die Folgerechtsvergütung zusteht. Das Erbrecht der Mitgliedstaaten sollte von dieser Richtlinie unberührt bleiben. Die Rechtsnachfolger des Urhebers müssen jedoch ... das Folgerecht nach dem Tod des Urhebers in vollem Umfang in Anspruch nehmen können."

12. Was die Sachvorschriften der Richtlinie anbelangt, verlangt Art. 1 Abs. 1, dass „[d]ie Mitgliedstaaten ... zugunsten des Urhebers des Originals eines Kunstwerks ein Folgerecht [vorsehen], das als unveräußerliches Recht konzipiert ist, auf das der Urheber auch im Voraus nicht verzichten kann; dieses Recht gewährt einen Anspruch auf Beteiligung am Verkaufspreis aus jeder Weiterveräußerung nach der ersten Veräußerung durch den Urheber". 
13. Die anzuwendenden Sätze werden in Art. 4 Abs. 1 einheitlich mit geringem Beurteilungsspielraum gemäß Art. 4 Abs. 2 und 3 festgesetzt.

14. Nach Art. 6 Abs. 1 ist „[d]ie Folgerechtsvergütung ... nach Artikel $1 \ldots$ an den Urheber des Werks und, vorbehaltlich des Artikels 8 Absatz 2, nach seinem Tod an seine Rechtsnachfolger zu zahlen".6

15. Art. 8 sieht insbesondere vor:

"(1) Die Schutzdauer des Folgerechts entspricht [einer Dauer, die das Leben des Urhebers und siebzig Jahre nach seinem Tod umfasst, unabhängig von dem Zeitpunkt, zu dem das Werk erlaubterweise der Öffentlichkeit zugänglich gemacht worden ist $\left.{ }^{7}\right]$.

(2) Abweichend von Absatz 1 brauchen die Mitgliedstaaten, die das Folgerecht am [Zeitpunkt des Inkrafttretens nach Artikel 13] nicht anwenden, während eines Zeitraums, der spätestens am 1. Januar 2010 abläuft, ein Folgerecht zugunsten der nach dem Tod des Künstlers anspruchsberechtigten Rechtsnachfolger nicht anzuwenden.

(3) Ein Mitgliedstaat, auf den Absatz 2 Anwendung findet, verfügt erforderlichenfalls über einen zusätzlichen Zeitraum von höchstens zwei Jahren, um die Wirtschaftsteilnehmer in diesem Mitgliedstaat in die Lage zu versetzen, sich unter Wahrung ihrer wirtschaftlichen Lebensfähigkeit allmählich an das Folgerechtssystem anzupassen, bevor dieses Recht zugunsten der nach dem Tod des Künstlers anspruchsberechtigten Rechtsnachfolger angewandt werden muss...."

16. Gemäß Art. 12 sind die Mitgliedstaaten verpflichtet, die Richtlinie vor dem 1. Januar 2006 umzusetzen, und Art. 13 legt als Tag ihres Inkrafttretens den Tag ihrer Veröffentlichung im Amtsblatt der Europäischen Gemeinschaften, d. h. den 13. Oktober 2001, fest.

\section{Französisches Recht}

17. In den französischen Rechtsvorschriften ist das Folgerecht seit 1920 vorgesehen. ${ }^{8}$ Die maßgebliche Vorschrift wurde jedoch 2006 zur vollständigen Anpassung an die Richtlinie geändert. ${ }^{9}$ Art. L.122-8 des Code de la propriété intellectuelle (Gesetz über das geistige Eigentum) sieht nunmehr vor:

„Den Urhebern des Originals eines Werks der bildenden Künste, die Angehörige eines Mitgliedstaats der Europäischen Gemeinschaft oder eines Vertragsstaats des Abkommens über den Europäischen Wirtschaftsraum sind, steht ein Folgerecht $z u$, bei dem es sich um ein unveräußerliches Recht auf Beteiligung am Erlös jeder Veräußerung des Werks nach seiner ersten Veräußerung durch den Urheber oder seinen Rechtsnachfolger handelt ..."

18. Weiter bestimmt Art. L.123-7:

"Nach dem Tod des Urhebers besteht das in Art. L.122-8 erwähnte Folgerecht zugunsten seiner Erben ... unter Ausschluss sämtlicher Vermächtnisnehmer und Rechtsnachfolger während des laufenden Kalenderjahrs und den folgenden 70 Jahren fort. ${ }^{\prime \prime 10}$

19. Ich werde diese Definition der Inhaber des Folgerechts nach dem Tod des Urhebers im Folgenden als „die umstrittene Regelung" bezeichnen.

20. Die Erbfolge bestimmt sich nach den Art. 734 f. des französischen Code civil und umfasst vier Erbordnungen. Die Rangfolge innerhalb jeder Ordnung hängt vom Verwandtschaftsgrad ab. Seitenverwandte fernerer Grade als des sechsten Grades sind nicht erbberechtigt. ${ }^{11}$

21. Die Art. 912 f. des Code civil teilen den Nachlass eines Verstorbenen in einen Teil, der kraft Gesetzes für bestimmte Erben vorgesehen ist, und einen Teil, über den durch Testament zugunsten von Vermächtnisnehmern verfügt werden kann. Grundsätzlich kann über den gesamten Nachlass testamentarisch verfügt werden, es sei denn, der Verstorbene wird von

8 Loi frappant d'un droit au profit des artistes les ventes publiques d'objets d'art (Gesetz zur Einziehung von Gebühren bei öffentlichen Kunstversteigerungen zugunsten der Künstler) vom 20.5.1920, aufgehoben und ersetzt durch die Loi $n^{\circ}$ 57-298 sur la propriété littéraire et artistique (Gesetz über literarische und künstlerische Urheberrechte) vom 11.3.1957.

9 Loi n ${ }^{\circ}$ 2006-961 relative au droit d'auteur et aux droits voisins dans la société de l'information (Gesetz über das Urheberrecht und verwandte Schutzrechte in der Informationsgesellschaft) vom 1. 8.2006.

10 Diese Vorschrift blieb unverändert, da die Laufzeit 1997 von 50 auf 70 Jahre verlängert worden war. Mit der Bezeichnung des Rechtsnachfolgers wird hier die französische Bezeichnung der ayants cause übersetzt, die vermutlich eine andere Bedeutung als die in der Richtlinie für den Rechtsnachfolger verwendete Bezeichnung des ayant droit hat, obwohl beide Ausdrücke im Französischen oft synonym verwendet werden.

11 Gemäß Art. 724 des Code civil erbt mangels eines gesetzlichen Erben oder eines Vermächtnisnehmers der Fiskus. 
Abkömmlingen oder dem Ehegatten einer bestehenden Ehe (oder, vor 2007, von Verwandten in gerade aufsteigender Linie) überlebt. Die umstrittene Regelung stellt damit eine Ausnahme von dieser allgemeinen Regel dar.

\section{Spanisches Recht}

22. Das Folgerecht wurde im spanischen Recht 1987 eingeführt ${ }^{12}$ und den Bestimmungen der Richtlinie durch das Gesetz 3/2008 angepasst. ${ }^{13}$ Anders als das französische Gesetz schließt das spanische Gesetz keine Personengruppe als Rechtsnachfolger des Urhebers eines Werks aus, sondern schreibt seit 1996 lediglich vor, dass das Folgerecht nur von Todes wegen übertragen werden kann.

\section{Sachverhalt, Verfahren und Vorlagefragen}

23. Im Jahr 1983 gründete Salvador Dalí die Fundació GalaSalvador Dalí ${ }^{14}$ (im Folgenden: Stiftung), „um in Spanien und in jedem anderen Staat das künstlerische, kulturelle und intellektuelle Werk des Malers, sein Eigentum und seine Rechte jedweder Art - seine Lebenserfahrung, sein Gedankengut, seine Vorhaben, Ideen und künstlerischen, intellektuellen und kulturellen Werke - sein Andenken und die universale Anerkennung des Genies seines Beitrags zu den Künsten, zur Kultur und zum zeitgenössischen Gedankengut zu präsentieren, zu wahren, zu verbreiten, zu zelebrieren, zu schützen und zu verteidigen". ${ }^{15}$

24. Dalí starb 1989 als Witwer; er hinterließ keine Kinder und Abkömmlinge, setzte aber in seinem Testament den spanischen Staat als Gesamtvermächtnisnehmer seiner Rechte des geistigen Eigentums ein, wobei er diesen leidenschaftlich aufforderte, seine Kunstwerke zu erhalten, zu verbreiten und zu schützen. Der Staat nahm das Vermächtnis an und übertrug die betreffenden Rechte dem Ministerium für Kultur zur ausschließlichen Verwaltung und Verwertung, das sie der Stiftung weiterübertrug.

25. 1997 beauftragte die Stiftung die spanische Verwertungsgesellschaft Visual Entidad de Gestión de Artistas Plásticos (im Folgenden: VEGAP), deren Mitglied sie ist, exklusiv mit der weltweiten kollektiven Verwaltung und Einziehung von Vergütungen im Hinblick auf das Werk von Dalí. Die VEGAP beauf-

12 Art. 24 der Ley 22/1987 de Propiedad Intelectual (Gesetz über das geistige Eigentum) vom 11.11.1987, geändert durch das Real Decreto Legislativo 1/1996 por el que se apruebe el texto refundido de la Ley de Propiedad Intelectual, regularizando, aclarando y armonizando las disposiciones legales vigentes sobre la materia (Königliches Gesetzesdekret, durch das die kodifizierte Fassung des Gesetzes über das geistige Eigentum genehmigt wurde, das die auf diesem Gebiet geltenden rechtlichen Bestimmungen regelt, präzisiert und harmonisiert) vom 12.4.1996.

13 Art. 2 Abs. 1 und 6 der Ley 3/2008 relativa al derecho de participación en beneficio del autor de una obra de arte original (Gesetz über das Folgerecht des Urhebers des Originals eines Kunstwerks) vom 23.12.2008.

14 Gala war der Name, unter dem seine Frau, Elena Dmitrievna Diakonova, allgemein bekannt war. Sie starb 1982.

15 Vgl. http://www.salvador-dali.org/fundacio/es_historia.html. tragte ihre französische Schwestergesellschaft Auteurs dans les Arts Graphiques et Plastiques (im Folgenden: ADAGP), mit der sie durch einen gegenseitigen Vertretungsvertrag verbunden ist, mit der Verwaltung der Rechte am Werk von Dalí in Frankreich ab dem 17. Oktober 1997.

26. Seitdem erhebt die ADAGP für die Stiftung sämtliche für die Verwertung des Werks des Künstlers in Frankreich geschuldeten Beträge und leitet sie an die VEGAP weiter - mit Ausnahme von Folgerechtsvergütungen, die sie, zumindest vorerst, für Dalís seitenverwandte Erben erhob und an diese zahlte.

27. Am 28. Dezember 2005 erhoben die Stiftung und die VEGAP vor dem Tribunal de grande instance de Paris Klage gegen die ADAGP. Sie machten geltend, sowohl nach französischem als auch nach spanischem Kollisionsrecht unterliege die Nachfolge für Dalís beweglichen Nachlass spanischem Recht, da Dalí bei seinem Tod in Spanien wohnhafter spanischer Staatsangehöriger gewesen sei. Die Stiftung sei daher alleinige Anspruchsberechtigte für sämtliche Rechte an Dalís Werk, vor allem für das Folgerecht bei öffentlichen Versteigerungen. Sie beantragte, die ADAGP zu verurteilen, ihr über die VEGAP alle Folgerechtsvergütungen aus dem Verkauf von Dalís Werken seit dem 17. Oktober 1997 zu zahlen.

28. Wie aus den Verfahrensakten des vorlegenden Gerichts hervorgeht, hat die ADAGP seit Klageerhebung keine derartigen Folgerechtsvergütungen erhoben und ist bereit, diese an die Partei oder die Parteien zu zahlen, die das Tribunal de grande instance für anspruchsberechtigt erklärt. Vergütungen, die sie bereits an die sechs seitenverwandten Erben gezahlt habe, die sie für nach französischem Recht berechtigt gehalten habe, seien, so ihr Vortrag, von diesen gegebenenfalls wieder einzufordern. Sie hat daher den Erben als weiteren Beteiligten den Streit verkündet, von denen jedoch keiner eine Stellungnahme abgegeben hat.

29. Das Tribunal de grande instance stellt fest, dass Frankreich ein Folgerecht nur zugunsten der gesetzlichen Erben beibehalten habe, während die Richtlinie besage, dass die Folgerechtsvergütung nach dem Tod des Künstlers an "seine Rechtsnachfolger" zu zahlen sei. Es fragt sich, ob die Richtlinie dies entweder als Regelfall oder als Ausnahme im Sinne des Art. 8 gestattet.

30. Es hat dem Gerichtshof daher folgende Fragen zur Vorabentscheidung vorgelegt:

Kann Frankreich nach Erlass der Richtlinie vom 27. September 2001 ein den Erben unter Ausschluss von Vermächtnisnehmern oder Rechtsnachfolgern vorbehaltenes Folgerecht beibehalten?

Gestatten die Übergangsbestimmungen des Art. 8 Abs. 2 und 3 der Richtlinie vom 27. September 2001 Frankreich eine Ausnahmeregelung? 
31. Schriftliche Erklärungen haben eingereicht die Stiftung und VEGAP, die französische, die italienische und die spanische Regierung sowie die Kommission. In der mündlichen Verhandlung haben die Stiftung, die französische und die spanische Regierung sowie die Kommission Stellung genommen.

\section{Würdigung}

32. Das vorlegende Gericht möchte im Wesentlichen wissen, ob die Richtlinie insbesondere im Hinblick auf Art. 6 Abs. 1 und Art. 8 Abs. 2 und 3 dahin auszulegen ist, dass es Frankreich gestattet ist, ein Folgerecht beizubehalten, das nach dem Tod des Urhebers dessen gesetzlichen Erben unter Ausschluss von Vermächtnisnehmern oder Rechtsnachfolgern vorbehalten ist.

33. Bevor ich jedoch auf diese Fragen eingehe, halte ich es für nützlich, bestimmte Gesichtspunkte zu prüfen, die die Anwendbarkeit der Richtlinie unter Umständen wie denen des Ausgangsverfahrens und, der Stellungnahme der spanischen Regierung zufolge, sogar die Zulässigkeit der Vorlagefragen berühren.

34. Erstens ist festzustellen, dass sich im Ausgangsverfahren Privatpersonen gegenüberstehen und Frankreich als Mitgliedstaat, an den die Richtlinie gerichtet ist, nicht als Partei beteiligt ist. Zweitens betrifft das Ausgangsverfahren zumindest teilweise Beträge, die möglicherweise einerseits vor Erlass der Richtlinie und andererseits nach ihrem Erlass, aber vor Ablauf ihrer Umsetzungsfrist erhoben worden sind. Drittens berufen sich die Kläger des Ausgangsverfahrens nicht auf Unvereinbarkeit des französischen Rechts mit der Richtlinie in irgendeiner Weise, sondern auf die Anwendbarkeit des spanischen anstelle des französischen Rechts zur Bestimmung der Inhaber des Folgerechts.

\section{„Unmittelbare horizontale Wirkung“}

35. Aus einer ständigen Rechtsprechung ergibt sich, dass eine Richtlinie nicht selbst Verpflichtungen für einen Einzelnen begründen kann, so dass ihm gegenüber eine Berufung auf die Richtlinie als solche nicht möglich ist und sogar eine klare, genaue und unbedingte Richtlinienbestimmung, mit der dem Einzelnen Rechte gewährt oder Verpflichtungen auferlegt werden sollen, im Rahmen eines Rechtsstreits, in dem sich ausschließlich Private gegenüberstehen, nicht als solche Anwendung finden kann. ${ }^{16}$ Da sich im vorliegenden Ausgangsverfahren in der Tat Private gegenüberstehen, schließt diese Grundregel anscheinend jegliche Berufung auf die Unvereinbarkeit des französischen Rechts mit der Richtlinie aus.

36. Aus dem Vorabentscheidungsersuchen und den nationalen Verfahrensakten, die dem Gerichtshof übermittelt worden sind, scheint jedoch klar hervorzugehen, dass sich die Stiftung

16 Vgl. zuletzt Urteil vom 16.7.2009, Mono Car Styling (C-12/08, Slg. 2009, I-0000, Randnr. 59). und die VEGAP im Ausgangsverfahren gegen die ADAGP oder Dalís Erben nicht auf die Richtlinie berufen wollen. Vielmehr hat das Tribunal de grande instance die Frage offensichtlich von Amts wegen aufgeworfen, und die Stiftung und die VEGAP haben nur in ihren Stellungnahmen vor dem Gerichtshof zu dem auf diese Weise aufgeworfenen Thema vorgebracht, die angefochtene Regelung sei mit der Richtlinie unvereinbar.

37. Unter diesen Umständen scheint mir der fraglichen Rechtsprechung tatsächlich im Hinblick auf den Sachverhalt keine Bedeutung zuzukommen. Obwohl der Gerichtshof die allgemeine Feststellung getroffen hat, dass eine Richtlinienvorschrift in einem Verfahren zwischen Privaten keine Anwendung finden kann, beruht diese Feststellung darauf, dass eine private Partei sich nicht auf die Bestimmungen einer Richtlinie berufen kann, um einen Anspruch gegen eine andere private Partei geltend zu machen oder ihr eine Verpflichtung aufzuerlegen. Diese Überlegung gilt nicht, wenn das nationale Gericht die Frage von Amts wegen aufwirft.

38. Nach der Rechtsprechung ist mangels einer einschlägigen Gemeinschaftsregelung (jetzt EU-Recht) die Bestimmung der zuständigen Gerichte und die Ausgestaltung von Verfahren, die den Schutz der dem Bürger aus dem EU-Recht erwachsenden Rechte gewährleisten sollen, Sache der innerstaatlichen Rechtsordnung der einzelnen Mitgliedstaaten, wobei diese Verfahren nicht weniger günstig gestaltet sein dürfen als bei entsprechenden Klagen, die nur innerstaatliches Recht betreffen (Äquivalenzgrundsatz), und die Ausübung der durch die Gemeinschaftsrechtsordnung verliehenen Rechte nicht praktisch unmöglich machen oder übermäßig erschweren dürfen (Effektivitätsgrundsatz). Daher steht das EU-Recht einer innerstaatlichen Vorschrift entgegen, die diese beiden Grundsätze missachtet, indem sie ein nationales Gericht davon abhält, von Amts wegen zu prüfen, ob eine Maßnahme des innerstaatlichen Rechts mit einer Bestimmung des EU-Rechts vereinbar ist. Es verlangt jedoch nicht, dass das nationale Gericht eine solche Frage von Amts wegen aufwirft, wenn keiner dieser Grundsätze in Rede steht. ${ }^{17}$

39. Zweifellos kann das EU-Recht ein nationales Gericht nicht daran hindern, von Amts wegen (wie im vorliegenden Fall geschehen) eine Frage der Vereinbarkeit innerstaatlichen Rechts mit den Vorschriften einer EU-Richtlinie aufzuwerfen. Vielmehr ist die Verpflichtung der nationalen Gerichte, innerstaatliches Recht so weit wie möglich anhand des Wortlauts und des

17 Diese hier zusammengefasste wohlbekannte Rechtsprechung begann mit den Urteilen vom 14.12.1995, Peterbroeck (C-312/93, Slg. 1995, I-4599) und Van Schijndel und van Veen (C-430/93 und C-431/93, Slg. 1995, I-4705), und wurde zuletzt im Urteil vom 7.6.2007, Van der Weerd u. a. (C-222/05 bis C-225/05, Slg. 2007, I-4233, Randnrn. 28 bis 42), dargelegt. Vgl. auch Schlussanträge des Generalanwalts Poiares Maduro in der letztgenannten Rechtssache, Nrn. 13 bis 41 . 
Zwecks der betreffenden Richtlinie auszulegen, um das in dieser festgelegte Ergebnis zu erreichen ${ }^{\mathbf{1 8}}$, ein positiver Ansporn, solche Fragen aufzuwerfen.

40. Im vorliegenden Fall bestimmt sich nach französischem Recht, ob das Tribunal de grande instance berechtigt ist, um Vorabentscheidung über die Vereinbarkeit der umstrittenen Regelung mit der Richtlinie zu ersuchen und die betreffende Entscheidung durchzusetzen. Sollte sich die Regelung als unvereinbar mit der Richtlinie erweisen, wäre sie daher voraussichtlich unanwendbar, da ihr ausdrücklicher Wortlaut kaum in dem Sinne ausgelegt werden könnte, dass Vermächtnisnehmer erfasst würden, und die Verpflichtung, nationales Recht in Übereinstimmung mit EU-Recht auszulegen, keine Auslegung des nationalen Gesetzes contra legem begründen kann. ${ }^{19}$

41. Es wurde jedoch nicht vorgetragen, dass das Tribunal de grande instance unzuständig sei, um Vorabentscheidung zu ersuchen oder alles Erforderliche zu tun, um die betreffende Entscheidung durchzusetzen. Ich werde im Folgenden davon ausgehen, dass es zuständig ist und die Entscheidung des Gerichtshofs in angemessener Weise durchsetzen kann.

\section{Zeitliche Anwendbarkeit der Richtlinie}

42. Die ADAGP erhebt seit dem 17. Oktober 1997 Folgerechtsvergütungen für Verkäufe von Dalís Werken. Die Richtlinie trat am 13. Oktober 2001 in Kraft, und die Mitgliedstaaten waren verpflichtet, die erforderlichen Umsetzungsmaßnahmen vor dem 1. Januar 2006 (vorbehaltlich bestimmter zeitlicher Ausnahmen gemäß Art. 8 Abs. 2 und 3, die Gegenstand der zweiten Frage des nationalen Gerichts sind, bis zum 1. Januar 2010 oder 1. Januar 2012) zu treffen.

43. Für die Zeit ab dem 1. Januar 2006 ist daher die Auslegung der Richtlinie maßgebend. Für die Zeit vor dem 13. Oktober 2001 oder die Zeit zwischen den beiden Daten kann ihr jedoch keine unmittelbare Bedeutung zukommen.

44. Obwohl die Mitgliedstaaten während der Umsetzungsfrist einer Richtlinie davon absehen müssen, Maßnahmen zu treffen, die den vorgeschriebenen Zweck ernsthaft gefährden würden, müssen sie ihre Gesetzgebung nicht vor Ablauf der Frist anpassen. Im vorliegenden Fall wurde die umstrittene Regelung während der Umsetzungsfrist nicht abgeändert.

45. Was die Verpflichtung zur richtlinienkonformen Auslegung anbelangt, besteht erst mit Ablauf der Umsetzungsfrist eine Verpflichtung der nationalen Gerichte, das innerstaatliche

18 Vgl. zuletzt Urteil Mono Car Styling (Fn. 16), Randnrn. 60 f.

19 Vgl. Urteil Mono Car Styling (Fn. 16 ), Randnr. 61 und die dort angeführte Rechtsprechung.
Recht (so weit wie möglich) richtlinienkonform auszulegen. ${ }^{20}$ In der Zwischenzeit müssen sie lediglich (abermals so weit wie möglich) davon absehen, das innerstaatliche Recht in einer Weise auszulegen, die die künftige Erreichung des mit der Richtlinie verfolgten Ziels nach Ablauf der Umsetzungsfrist ernsthaft gefährden würde. ${ }^{21}$

46. Wie festgestellt, lässt sich der eindeutige Wortlaut der umstrittenen Regelung jedoch kaum anders auslegen. Daher scheint die Einschränkung durch den Ausdruck "so weit wie möglich" in der Rechtsprechung des Gerichtshofs der Verpflichtung zur richtlinienkonformen Auslegung im vorliegenden Fall entgegenzustehen. Bei Unvereinbarkeit der umstrittenen Regelung mit der Richtlinie wäre es daher offensichtlich nur möglich, die Regelung nicht anzuwenden ${ }^{22}$, und diese Verpflichtung könnte nur für die Zeit nach Ablauf der Umsetzungsfrist entstehen. Wäre die umstrittene Regelung in diesem Zeitraum unanwendbar, stellte sich offensichtlich die weitere Frage, ob sie im Hinblick auf frühere Zeiträume noch anwendbar wäre. Dies wäre aber ausschließlich eine Sache des französischen Rechts und nicht des EU-Rechts.

\section{Anwendbares Recht}

47. Das Hauptargument der Stiftung und der VEGAP sowohl im Ausgangsverfahren als auch vor dem Gerichtshof sowie der spanischen Regierung im Verfahren vor dem Gerichtshof geht dahin, dass das spanische und nicht das französische Recht zu bestimmen habe, wer Rechtsnachfolger von Salvador Dalí nach dessen Tod sei, da der Nachlass an seinem beweglichen Vermögen dem spanischem Erbrecht unterliege. Daher stelle sich nicht die Frage der Vereinbarkeit der umstrittenen Regelung mit der Richtlinie. Die spanische Regierung führt ferner aus, die Vorlagefragen seien unzulässig, da sie zur Entscheidung des Rechtsstreits im Ausgangsverfahren nicht erforderlich seien.

48. Zwar kann die Entscheidung über eine Vorlagefrage eines nationalen Gerichts abgelehnt werden, wenn die erbetene Auslegung des EU-Rechts offensichtlich in keinem Zusammenhang mit dem Sachverhalt oder dem Gegenstand des Ausgangsrechtsstreits steht ${ }^{23}$, doch kann dies meines Erachtens im vorliegenden Fall nicht angenommen werden. Andernfalls müsste der Gerichtshof über innerstaatliches Recht entschei-

20 Vgl. Urteile vom 18.12.1997, Inter-Environnement Wallonie (C-129/96, Slg. 1997, I-7411, insbesondere Randnrn. 43 bis 45), und vom 4.7.2006, Adeneler u. a. (C-212/04, Slg. 2006, I-6057, Randnrn. 114 und 115).

21 Vgl. Urteile Adeneler (Fn. 20), Randnr. 123, und vom 23.4.2009, VTB-VAB und Galatea (C-261/07 und C-299/07, Slg. 2009, I-0000, Randnr. 39).

22 Vgl. zuletzt Urteil vom 27.10.2009, ČEZ (C-115/08, Slg. 2009, I-0000, Randnr. 140).

23 Vgl. zuletzt Urteil vom 1.10.2009, Compañía Española de Comercialización de Aceite u. a. (C-505/07, Slg. 2009, I-0000, Randnr. 26). 
den - die Bestimmung des im Erbfall anwendbaren Rechts ist bislang im Unionsrecht in keiner Weise geregelt - und würde damit seine Zuständigkeit überschreiten. ${ }^{24}$

49. Unter Umständen wie denjenigen des Ausgangsverfahrens scheint mir das nationale Gericht jedoch zum einen einer Antwort auf das Problem zu bedürfen, ob die Frage, wer Inhaber des Folgerechts nach dem Tod des Künstlers ist, dem Recht unterliegt, nach dem die Folgerechtsvergütungen erhoben werden, oder dem Recht, dem die Rechtsnachfolge für den Nachlass des Künstlers unterliegt. Nur im letztgenannten Fall muss es entscheiden, welchem Recht diese Rechtsnachfolge unterliegt - eine Frage, die außerhalb der Zuständigkeit des Gerichtshofs liegt. Zum anderen kann der Gerichtshof aufzeigen, ob die Richtlinie eine Orientierungshilfe für die Ausgangsfrage gibt.

50. Der Begriff des Rechtsnachfolgers des Urhebers ist in der Richtlinie nirgendwo definiert. Der 27. Erwägungsgrund überlässt seine Definition implizit, aber gleichwohl eindeutig dem Recht der Mitgliedstaaten und verweist insbesondere auf deren Erbrecht. Wie sich aus der Entstehungsgeschichte der Richtlinie ergibt, war dies im Rechtssetzungsprozess auch die Auffassung der Kommission und des Rates. ${ }^{25}$ Hingegen war das Parlament bei seinem Änderungsvorschlag zum Entwurf der Vorschrift überzeugt, dass die Bestimmung, wem das Folgerecht nach dem Tod des Urhebers zustehe, nach dem Subsidiaritätsgrundsatz den einzelstaatlichen Rechtsordnungen überlassen bleiben müsse und das Erbrecht unangetastet bleiben solle. ${ }^{26}$ Ich möchte hinzufügen, dass die Richtlinie, wäre eine Harmonisierung der Kollisionsnormen im Bereich des Erbrechts beabsichtigt gewesen, nicht, wie geschehen, einfach auf Art. 95 EG ${ }^{27}$

24 Die Haager Konferenz für Internationales Privatrecht hat ein am 1.8.1989 geschlossenes Übereinkommen über das in Erbrechtsfällen anwendbare Recht entworfen - aber von den Mitgliedstaaten der Europäischen Union haben dieses nur Luxemburg und die Niederlande unterzeichnet und nur die Niederlande ratifiziert. Zudem veröffentlichte die Kommission einen Monat vor der mündlichen Verhandlung in der vorliegenden Rechtssache einen Vorschlag für eine Verordnung des Europäischen Parlaments und des Rates über die Zuständigkeit, das anzuwendende Recht, die Anerkennung und die Vollstreckung von Entscheidungen und öffentlichen Urkunden in Erbsachen sowie zur Einführung eines Europäischen Nachlasszeugnisses (KOM[2009] 154 endg. vom 14.10.2009) - aber dieser Vorschlag ist natürlich noch weit davon entfernt, rechtsverbindlich zu sein. Nach den Art. 3 f. des Haager Übereinkommens und $16 \mathrm{f}$. des Vorschlags der Kommission unterläge, ihre Anwendbarkeit unterstellt, die Rechtsnachfolge für den Nachlass von Salvador Dalí dem spanischen Recht.

25 Vgl. insbesondere die Begründung des Rates vom 5.6.2000 für seinen gemeinsamen Standpunkt vom 22.5.2000 (7484/00 ADD 1), Randnr. 23, und die Stellungnahme der Kommission vom 24.1.2001 zu den Abänderungen des Europäischen Parlaments an dem gemeinsamen Standpunkt des Rates (KOM[2001] 47 endg.), Nr. 3.1.2 Buchst. b.

26 Vgl. den Bericht des Parlaments für die erste Lesung vom 3.2.1997 (Dokument A4-0030/97), Begründung, Nr. IV Abschnitt A Abs. 2, und den Bericht für die zweite Lesung vom 29.11.2000 (Dokument A5-0370/2000), Begründung, Abschnitt III Abs. 8.

27 Vgl. jetzt Art. 114 AEUV. hätte gestützt werden können, sondern entsprechend dem in Fn. 24 erwähnten Vorschlag auf die Art. 61 EG und 67 EG $^{28}$ hätte Bezug nehmen müssen.

51. Wenn vor einem Gericht des Mitgliedstaats, in dem eine Folgerechtsvergütung erhoben worden ist, darüber gestritten wird, wer nach dem Tod des Urhebers eines Werks Inhaber des Folgerechts ist, muss dieses Gericht folglich die nach seinem innerstaatlichen Recht dafür maßgebenden Regeln anwenden. Soweit nicht anders geregelt, wird dies das materielle Recht der Rechtsordnung sein, der deren Kollisionsrecht zufolge das Erbrecht unterliegt.

52. Aus der Tatsache, dass die Richtlinie innerstaatliches Recht eindeutig unangetastet lassen will und die Bestimmung der Anspruchsberechtigten nach dem Tod des Künstlers nicht ausschließlich mit dem Recht verknüpft, dem die Rechtsnachfolge für seinen Nachlass unterliegt, ergibt sich meines Erachtens, dass sie es einem Mitgliedstaat nicht untersagt, eine spezifische materiell-rechtliche Vorschrift zu erlassen, die sich ganz oder zum Teil über das Kollisionsrecht hinwegsetzt, nach dem sich dieses Recht andernfalls bestimmen würde.

53. Dieser Schluss scheint auch am ehesten Art. 14ter der Berner Übereinkunft zu entsprechen, nach dem das Folgerecht nach dem Tod des Urhebers den „von den innerstaatlichen Rechtsvorschriften dazu berufenen Personen oder Institutionen" zufällt, wobei dieser Wortlaut offensichtlich weiter ist als ein Verweis auf erbrechtliche Regelungen im weitesten Sinne.

54. Das Tribunal de grande instance muss daher feststellen, ob die umstrittene Regelung eine solche vorrangige Regelung darstellt und, falls nicht, welches Erbrecht nach dem anwendbaren Kollisionsrecht maßgebend ist.

55. Sollte diese Prüfung ergeben, dass die umstrittene Regelung im Ausgangsverfahren anwendbar ist, muss auf die Frage ihrer Vereinbarkeit mit der Richtlinie eingegangen werden.

\section{Frage 1}

56. Gestattet es die Richtlinie einem Mitgliedstaat, in seinen innerstaatlichen Rechtsvorschriften den Kreis der Rechtsnachfolger des Künstlers in der in der umstrittenen Regelung vorgesehenen Weise zu beschränken?

57. Aus ähnlichen Gründen wie denen, die ich oben bei Prüfung des den Mitgliedstaaten bei Bestimmung des anwendbaren Rechts eingeräumten Beurteilungsspielraums erörtert habe, scheint mir diese Frage zu bejahen zu sein.

58. Die Richtlinie definiert den Begriff des Rechtsnachfolgers des Künstlers nach dessen Tod nicht. Sie überlässt diese Bestimmung dem innerstaatlichen Recht und damit vor allem (obgleich

28 Vgl. jetzt Art. 67 AEUV. 
nicht notwendigerweise ausschließlich) dem innerstaatlichen Erbrecht. Unterschiede zwischen innerstaatlichen Rechtsordnungen, die sich voraussichtlich nicht nachteilig auf den Binnenmarkt auswirken, können bestehen bleiben. ${ }^{29}$ Es gibt somit keinen einheitlichen Kreis der Rechtsnachfolger, und die Mitgliedstaaten können jedwede Definition festlegen oder beibehalten, die voraussichtlich keine derartigen Auswirkungen hat.

59. Wie aus dem neunten Erwägungsgrund hervorgeht ${ }^{30}$, war es Hauptzweck der Richtlinie zu verhindern, dass sich die Veräußerungen von Kunstwerken zum Nachteil von Auktionshäusern oder anderen Kunsthändlern in den Mitgliedstaaten, die den ursprünglichen Künstler (oder einen Rechtsnachfolger) an dem durch die Wertsteigerung der Kunstwerke erzielten Gewinn beteiligen wollten, in Mitgliedstaaten konzentrierten, in denen das Folgerecht entweder nicht galt oder weniger beschwerend war.

60. Diese Situation bestand vor Erlass der Richtlinie und beruhte auf der Abneigung der Verkäufer, auf einen Teil des mit dem Verkauf eines Kunstwerks erzielten Erlöses zu verzichten. Ich bin mit der Kommission der Meinung, dass nach Erlass der Richtlinie die Wahrscheinlichkeit äußerst gering ist, dass Verkäufer unverzüglich den Mitgliedstaat wählen werden, in dem sie unter Bezugnahme auf die Anspruchsberechtigten der Folgerechtsvergütung verkaufen - ein Faktor, der den Preis nicht beeinflusst und dem Verkäufer sogar unbekannt sein kann - und sich voraussichtlich nicht nachteilig auf den Binnenmarkt auswirken wird. Insofern bin ich von dem Vorschlag der spanischen Regierung in der mündlichen Verhandlung nicht überzeugt eher befremdet -, dass sich der Handel in Mitgliedstaaten verlagern könnte, in denen es keinen Rechtsnachfolger des Künstlers gibt. Selbst wenn eine innerstaatliche Rechtsordnung keine bestimmte Regelung trifft, geht der Nachlass eines verstorbenen Künstlers offensichtlich immer auf irgendjemanden über, und sei es auch auf den Fiskus als ultimus haeres.

61. Entsprechend würde ich auch dem Vorbringen der Stiftung und der VEGAP sowie der spanischen Regierung entgegentreten, der Begriff des Rechtsnachfolgers eines verstorbenen Künstlers müsse alle erfassen, die dem anwendbaren Erbrecht zufolge Rechtsnachfolger seien, und könne sich nicht in anspruchsberechtigte und nicht anspruchsberechtigte Rechtsnachfolger aufteilen.

62. Wenn die umstrittene Regelung den erbrechtlichen Kollisionsnormen vorgeht, kann es keinen Grund geben, warum sie nicht Personen ausschließen können soll, die dem Erbrecht zufolge Rechtsnachfolger hätten sein können. Jedenfalls kann sich der Ausschluss bestimmter Personen nicht nachteilig auf den Binnenmarkt auswirken.

29 13. Erwägungsgrund.

30 Siehe oben, Nr. 8.
63. Nichts anderes gilt, falls die umstrittene Regelung selbst eine materielle erbrechtliche Vorschrift darstellt. Die Freiheit eines Einzelnen, über seinen Nachlass zu verfügen, mag in den einzelnen Rechtsordnungen unterschiedlich geregelt sein, und verschiedene Regelungen oder Mechanismen können die Möglichkeit einschränken, Zuwendungen beispielsweise an Personen außerhalb des Kreises der gesetzlichen Erben oder der Abkömmlinge und/oder einen überlebenden Ehegatten zu machen. Die in der umstrittenen Regelung vorgenommene Unterscheidung fällt offensichtlich in diesen Bereich und gehört somit zu den zulässigen Wahlmöglichkeiten des innerstaatlichen Erbrechts, auf das sich die Richtlinie zur Bestimmung der Inhaber des Folgerechts nach dem Tod des Künstlers bezieht.

64. In der mündlichen Verhandlung wurde erörtert, ob die Mitgliedstaaten nicht bei der Bestimmung des Rechtsnachfolgers die Erbrechtsregelungen des jeweils anderen im Sinne einer loyalen Zusammenarbeit oder vielleicht eines guten Einvernehmens der Mitgliedstaaten zu beachten hätten, auch wenn die Richtlinie diese Bestimmung dem innerstaatlichen Recht überlasse. Ich fürchte jedoch, dass eine solche Auffassung einer Harmonisierung des Erbrechts oder der Kollisionsnormen "durch die Hintertür" gefährlich nahekäme, die - sowohl angesichts der Rechtsgrundlage der Richtlinie als auch des ausdrücklichen gesetzgeberischen Willens - über die Reichweite der Richtlinie hinausginge.

65. Aus beiden Gründen ergibt sich, dass sich die Rolle der Richtlinie darauf beschränkt, Beeinträchtigungen des wettbewerblich geprägten Umfelds im Binnenmarkt zu beseitigen. Gegenseitige Anerkennung - in diesem Fall die Anerkennung der Bestimmung des Rechtsnachfolgers eines verstorbenen Künstlers gemäß dem Recht des Mitgliedstaats, dessen Erbrecht auf den Nachlass des Künstlers anzuwenden ist, durch den Mitgliedstaat, der die Folgerechtsvergütung erhebt, - ist ein lobenswertes Vorgehen. Ich glaube jedoch nicht, dass es der in Rede stehenden Richtlinie unterfällt. Der Hauptzweck der Richtlinie besteht darin, sicherzustellen, dass in der gesamten Union eine Folgerechtsvergütung erhoben wird. Sie soll jedoch nicht gewährleisten, dass das Recht genau denjenigen zugutekommt, die Rechtsnachfolger im Sinne eines bestimmten Erbrechts sind.

66. Die mit der umstrittenen Regelung getroffene Entscheidung ist eine politische Entscheidung, über die man immer streiten kann. ${ }^{31}$ Sie ist jedoch meines Erachtens eine Entscheidung, die sich vollkommen im Rahmen des dem Mitgliedstaat

31 Man könnte sich z. B. fragen, warum eine solche Regel nur auf das Folgerecht und nicht etwa auf das Urheberrecht bei literarischen Werken anwendbar sein sollte (wobei die Antwort möglicherweise damit zusammenhängt, dass das Folgerecht, anders als das Urheberrecht bei literarischen Werken, unabtretbar und unveräußerlich ist). Die Debatte wäre im vorliegenden Fall wahrscheinlich besonders hitzig gewesen, wenn Salvador Dalí nicht von Erben des sechsten oder eines näheren Ranges überlebt worden wäre, so dass die Folgerechtsvergütung ohne Rücksicht darauf, dass sie nach Dalís ausdrücklichem Plan an den spanischen Staat gehen sollte, vom französischen Staat erhoben worden wäre. 
zustehenden Beurteilungsspielraums hält und die sich voraussichtlich nicht nachteilig auf den Binnenmarkt auswirkt. Die Richtlinie steht ihr daher nicht entgegen.

\section{Frage 2}

67. Falls der Gerichtshof meinem Vorschlag für eine Antwort auf die erste Frage zustimmt, erübrigt sich die Antwort auf die zweite Frage - ob die umstrittene Regelung aufgrund der fakultativen und übergangsweisen Ausnahmen in Art. 8 Abs. 2 und 3 der Richtlinie beibehalten werden kann. Sollte gleichwohl eine Antwort erforderlich sein, kann diese sehr kurz sein.

68. Die Ausnahmen in Art. 8 in Verbindung mit Art. 13 der Richtlinie können ausdrücklich von den Mitgliedstaaten in Anspruch genommen werden, die am 13. Oktober 2001 kein Folgerecht kannten.

69. Da es in Frankreich ein Folgerecht gab, können die Ausnahmen nicht in Anspruch genommen werden.
70. Jedenfalls gestatten es diese Ausnahmen Mitgliedstaaten nur, das Folgerecht zugunsten der Rechtsnachfolger des Künstlers nicht anzuwenden; sie betreffen nicht die Anwendung nur auf einen beschränkten Kreis von Rechtsnachfolgern.

\section{Ergebnis}

71. Nach alledem schlage ich dem Gerichtshof vor, die vom Tribunal de grande instance de Paris zur Vorabentscheidung vorgelegten Fragen wie folgt zu beantworten:

Die Richtlinie 2001/84/EG des Europäischen Parlaments und des Rates über das Folgerecht des Urhebers des Originals eines Kunstwerks steht einer innerstaatlichen Regelung nicht entgegen, nach der das Folgerecht nach dem Tod des Urhebers unter Ausschluss von Vermächtnisnehmern oder Rechtsnachfolgern nur auf gesetzliche Erben übergeht.

\title{
III. EuGH, Urteil vom 15. April 2010 - C-518/ 08
}

\begin{abstract}
Art. 6 Abs. 1 der Richtlinie 2001/ 84/ EG des Europäischen Parlaments und des Rates vom 27. September 2001 über das Folgerecht des Urhebers des Originals eines Kunstwerks ist dahin auszulegen, dass er nicht einer innerstaatlichen Rechtsvorschrift wie der im Ausgangsverfahren fraglichen entgegensteht, nach der auf Folgerechtsvergütungen unter Ausschluss durch Testament eingesetzter Vermächtnisnehmer allein die gesetzlichen Erben des Künstlers Anspruch haben. Es ist daher Sache des vorlegenden Gerichts, für die Anwendung der innerstaatlichen Rechtsvorschrift, durch die Art. 6 Abs. 1 der Richtlinie 2001/ 84 umgesetzt wird, ordnungsgemäß alle einschlägigen Bestimmungen zu berücksichtigen, die Kollisionen von gesetzlichen Regelungen über den für das Folgerecht geltenden Erbanfall lösen sollen.
\end{abstract}

- In der Rechtssache C-518/ 08 betreffend ein Vorabentscheidungsersuchen nach Art. 234 EG, eingereicht vom Tribunal de grande instance de Paris (Frankreich) mit Entscheidung vom 29. Oktober 2008, beim Gerichtshof eingegangen am 27. November 2008, in dem Verfahren Fundación Gala-Salvador Dalí, Visual Entidad de Gestión de Artistas Plásticos (VEGAP) gegen Société des auteurs dans les arts graphiques et plastiques (ADAGP), Juan-Leonardo Bonet Domenech, Eulalia-María Bas Dalí, María del Carmen Domenech Biosca, Antonio Domenech Biosca, AnaMaría Busquets Bonet, Mónica Busquets Bonet erlässt DER GERICHTSHOF (Dritte Kammer) unter Mitwirkung des Kammerpräsidenten K. Lenaerts, der Richterin R. Silva de Lapuerta sowie der Richter E. Juhász, J. Malenovský (Berichterstatter) und D. Šváby, Generalanwältin: E. Sharpston, Kanzler: N. Nanchev, Verwaltungsrat, aufgrund des schriftlichen Verfahrens und auf die mündliche Verhandlung vom 12. November 2009, unter Berücksichtigung der Erklärungen - der Fundación Gala-Salvador Dalí und der Visual Entidad de Gestión de Artistas Plásticos (VEGAP), vertreten durch P.-F. Veil, avocat, - der französischen Regierung, vertreten durch $G$. de Bergues und $B$. Beaupère-Manokha als Bevollmächtigte, - der spanischen Regierung, vertreten durch M. Muñoz Pérez als Bevollmächtigten, - der italienischen Regierung, vertreten durch G. Palmieri als Bevollmächtigte im Beistand von W. Ferrante, avvocato dello Stato, - der Kommission der Europäischen Gemeinschaften, vertreten durch H. Krämer und C. Vrignon als Bevollmächtigte, nach Anhörung der Schlussanträge der Generalanwältin in der Sitzung vom 17. Dezember 2009 folgendes Urteil*:

1. Das Vorabentscheidungsersuchen betrifft die Auslegung von Art. 6 Abs. 1 und Art. 8 Abs. 2 und 3 der Richtlinie 2001/ 84/ EG des Europäischen Parlaments und des Rates vom 27. September 2001 über das Folgerecht des Urhebers des Originals eines Kunstwerks (ABI. L 272, S. 32).

Verfahrenssprache: Französisch. 
2. Dieses Ersuchen ergeht im Rahmen eines Rechtsstreits zwischen der Fundación Gala-Salvador Dalí und der Visual Entidad de Gestión de Artistas Plásticos (im Folgenden: VEGAP) einerseits und der Société des auteurs dans les arts graphiques et plastiques (im Folgenden: ADAGP) sowie Herrn Bonet Domenech, Frau Bas Dalí, Frau Domenech Biosca, Herrn Domenech Biosca, Frau Ana-María Busquets Bonet und Frau Mónica Busquets Bonet, die der Familie des Malers Salvador Dalí angehören, andererseits wegen Folgerechtsvergütungen, die bei Verkäufen von Kunstwerken Salvador Dalís eingenommen wurden.

\section{Rechtlicher Rahmen}

\section{Die Richtlinie 2001/84}

3. Die Erwägungsgründe 3 und 4 der Richtlinie 2001/ 84 lauten:

„(3) Das Folgerecht soll den Urhebern von Werken der bildenden Künste eine wirtschaftliche Beteiligung am Erfolg ihrer Werke garantieren. Auf diese Weise soll ein Ausgleich zwischen der wirtschaftlichen Situation der bildenden Künstler und der Situation der anderen Kunstschaffenden hergestellt werden, die aus der fortgesetzten Verwertung ihrer Werke Einnahmen erzielen.

(4) Das Folgerecht ist Bestandteil des Urheberrechts und stellt ein wesentliches Vorrecht der Urheber dar. Um den Urhebern ein angemessenes und einheitliches Schutzniveau zu gewährleisten, ist die Einführung des Folgerechts in allen Mitgliedstaaten notwendig."

4. Die Erwägungsgründe 9 und 10 der Richtlinie 2001/ 84 lauten:

"(9) Das Folgerecht ist derzeit in den innerstaatlichen Rechtsvorschriften der meisten Mitgliedstaaten vorgesehen. Diese Rechtsvorschriften weisen - soweit sie bestehen - Unterschiede insbesondere in Bezug auf die erfassten Werke, die Anspruchsberechtigten, die Höhe des Satzes, die diesem Recht unterliegenden Transaktionen und die Berechnungsgrundlage auf. Die Anwendung oder Nichtanwendung eines solchen Rechts hat insofern erhebliche Auswirkungen auf die Wettbewerbsbedingungen im Binnenmarkt, als das Bestehen oder Nichtbestehen einer aus dem Folgerecht resultierenden Abführungspflicht ein Aspekt ist, der von jeder an dem Verkauf eines Kunstwerks interessierten Person in Betracht zu ziehen ist. Daher ist dieses Recht einer der Faktoren, die zu Wettbewerbsverzerrungen und Handelsverlagerungen in der Gemeinschaft beitragen.

(10) Diese Unterschiede hinsichtlich des Bestehens des Folgerechts und seiner Anwendung durch die Mitgliedstaaten haben unmittelbare negative Auswirkungen auf das reibungslose Funktionieren des Binnenmarkts im Sinne von Artikel 14 des Vertrags, soweit er Kunstwerke betrifft. Artikel 95 [EG] ist daher die geeignete Rechtsgrundlage."
5. Die Erwägungsgründe 13 bis 16 der Richtlinie 2001/ 84 lauten:

"(13) Bestehende rechtliche Unterschiede sollten beseitigt werden, soweit sie den Binnenmarkt in seiner Funktion beeinträchtigen, und es sollte verhindert werden, dass neue Unterschiede auftreten. Unterschiede hingegen, die sich voraussichtlich nicht nachteilig auf den Binnenmarkt auswirken, können bestehen bleiben bzw. müssen nicht verhindert werden.

(14) Eine Voraussetzung für das reibungslose Funktionieren des Binnenmarktes sind Wettbewerbsbedingungen, die keine Verzerrungen aufweisen. Unterschiede zwischen den nationalen Rechtsvorschriften zum Folgerecht lassen Wettbewerbsverzerrungen und Handelsverlagerungen in der Gemeinschaft entstehen und führen - je nachdem, wo die Werke verkauft werden - zu einer Ungleichbehandlung der Künstler. Die zur Prüfung stehende Frage weist somit transnationale Aspekte auf, die sich durch Maßnahmen der Mitgliedstaaten nicht befriedigend regeln lassen. Eine Untätigkeit der Gemeinschaft wäre unvereinbar mit dem Gebot des Vertrags, Wettbewerbsverzerrungen und Ungleichbehandlung entgegenzuwirken.

(15) In Anbetracht des Ausmaßes der Unterschiede zwischen den nationalen Bestimmungen ist es daher erforderlich, Harmonisierungsmaßnahmen zu erlassen, um Disparitäten zwischen den Rechtsvorschriften der Mitgliedstaaten in den Bereichen zu beheben, in denen diese Disparitäten die Schaffung oder die Aufrechterhaltung von Wettbewerbsverzerrungen zur Folge haben könnten. Eine Harmonisierung sämtlicher Bestimmungen der Mitgliedstaaten zum Folgerecht erscheint jedoch nicht erforderlich; damit so viel Spielraum wie möglich für einzelstaatliche Entscheidungen bleibt, genügt es, nur diejenigen einzelstaatlichen Vorschriften zu harmonisieren, die sich am unmittelbarsten auf das Funktionieren des Binnenmarkts auswirken.

(16) Diese Richtlinie entspricht daher in ihrer Gesamtheit dem Subsidiaritäts- und Verhältnismäßigkeitsprinzip nach Artikel 5 des Vertrags."

\section{Der 27. Erwägungsgrund der Richtlinie 2001/ 84 lautet:}

„Es erscheint angezeigt, unter Wahrung des Subsidiaritätsprinzips den Kreis der Personen zu bestimmen, denen die Folgerechtsvergütung zusteht. Das Erbrecht der Mitgliedstaaten sollte von dieser Richtlinie unberührt bleiben. Die Rechtsnachfolger des Urhebers müssen jedoch, zumindest nach Ablauf des oben genannten Übergangszeitraums, das Folgerecht nach dem Tod des Urhebers in vollem Umfang in Anspruch nehmen können."

7. Art. 1 ("Gegenstand des Folgerechts") Abs. 1 der Richtlinie 2001/ 84 bestimmt: 
„Die Mitgliedstaaten sehen zugunsten des Urhebers des Originals eines Kunstwerks ein Folgerecht vor, das als unveräußerliches Recht konzipiert ist, auf das der Urheber auch im Voraus nicht verzichten kann; dieses Recht gewährt einen Anspruch auf Beteiligung am Verkaufspreis aus jeder Weiterveräußerung nach der ersten Veräußerung durch den Urheber."

8. Art. 6 („Anspruchsberechtigte“) Abs. 1 der Richtlinie 2001/ 84 sieht vor:

„Die Folgerechtsvergütung nach Artikel 1 ist an den Urheber des Werks und, vorbehaltlich des Artikels 8 Absatz 2, nach seinem Tod an seine Rechtsnachfolger zu zahlen."

9. Art. 8 ("Schutzdauer des Folgerechts") Abs. 1 bis 3 der Richtlinie 2001/ 84 bestimmt:

"1(1) Die Schutzdauer des Folgerechts entspricht der in Artikel 1 der Richtlinie 93/ 98/ EWG [des Rates vom 29. Oktober 1993 zur Harmonisierung der Schutzdauer des Urheberrechts und bestimmter verwandter Schutzrechte] vorgesehenen Schutzdauer [an Werken der Literatur und Kunst im Sinne des Art. 2 der Berner Übereinkunft, die das Leben des Urhebers und 70 Jahre nach seinem Tod umfasst]."

(2) Abweichend von Absatz 1 brauchen die Mitgliedstaaten, die das Folgerecht am [Zeitpunkt des Inkrafttretens nach Art. 13] nicht anwenden, während eines Zeitraums, der spätestens am 1. Januar 2010 abläuft, ein Folgerecht zugunsten der nach dem Tod des Künstlers anspruchsberechtigten Rechtsnachfolger nicht anzuwenden.

(3) Ein Mitgliedstaat, auf den Absatz 2 Anwendung findet, verfügt erforderlichenfalls über einen zusätzlichen Zeitraum von höchstens zwei Jahren, um die Wirtschaftsteilnehmer in diesem Mitgliedstaat in die Lage zu versetzen, sich unter Wahrung ihrer wirtschaftlichen Lebensfähigkeit allmählich an das Folgerechtssystem anzupassen, bevor dieses Recht zugunsten der nach dem Tod des Künstlers anspruchsberechtigten Rechtsnachfolger angewandt werden muss...."

10. Art. 12 („Umsetzung der Richtlinie“) Abs. 1 Unterabs. 1 der Richtlinie 2001/ 84 sieht vor:

„Die Mitgliedstaaten setzen die Rechts- und Verwaltungsvorschriften in Kraft, die erforderlich sind, um dieser Richtlinie vor dem 1. Januar 2006 nachzukommen. Sie setzen die Kommission unverzüglich davon in Kenntnis."

\section{Nationales Recht}

11. Durch die Loi $n^{\circ} 2006-961$ relative au droit d'auteur et aux droits voisins dans la société de l'information (Gesetz über das Urheberrecht und verwandte Schutzrechte in der Informationsgesellschaft) vom 1. August 2006 (JORF vom 3. August 2006, S. 11529), mit der die Richtlinie 2001/ 84 in das innerstaatliche französische Recht umgesetzt wurde, wurde Art. L. 122-8 des Code de la propriété intellectuelle (Gesetzbuch des geistigen Eigentums, im Folgenden: $\mathrm{CPI}$ ) wie folgt neu gefasst:

"Den Urhebern des Originals eines Werks der bildenden Künste, die Angehörige eines Mitgliedstaats der Europäischen Gemeinschaft oder eines Vertragsstaats des Abkommens über den Europäischen Wirtschaftsraum sind, steht ein Folgerecht $z u$, bei dem es sich um ein unveräußerliches Recht auf Beteiligung am Erlös jeder Veräußerung des Werks, an der als Verkäufer, Käufer oder Vermittler ein Vertreter des Kunstmarkts beteiligt ist, nach seiner ersten Veräußerung durch den Urheber oder seine Rechtsnachfolger handelt ..."

12. Art. L. 123-7 CPI, der bei der Umsetzung der Richtlinie 2001/ 84 nicht geändert wurde, bestimmt:

"Nach dem Tod des Urhebers besteht das in Art. L. 122-8 erwähnte Folgerecht zugunsten seiner Erben und, was den Nießbrauch gemäß Art. L. 123-6, angeht, seines Ehegatten unter Ausschluss sämtlicher Vermächtnisnehmer und Rechtsnachfolger während des laufenden Kalenderjahrs und den folgenden 70 Jahren fort."

\section{Ausgangsrechtsstreit und Vorlagefragen}

13. Der Maler Salvador Dalí starb am 23. Januar 1989 in Figueras (Spanien) und hinterließ fünf gesetzliche Erben. Mit letztwilliger Verfügung vom 20. September 1982 hatte er seine gesamten Immaterialgüterrechte dem spanischen Staat als Gesamtvermächtnisnehmer im Sinne des französischen Erbrechts vermacht. Diese Rechte werden von der Fundación Gala-Salvador Dalí verwaltet, die 1983 auf Initiative und unter Kontrolle des Malers als Stiftung spanischen Rechts gegründet worden war.

14. Die Fundación Gala-Salvador Dalí betraute 1997 die VEGAP, eine Gesellschaft spanischen Rechts, exklusiv und weltweit mit der kollektiven Verwaltung und Wahrnehmung der Urheberrechte am Werk von Salvador Dalí.

15. Die VEGAP ist vertraglich mit ihrer Schwestergesellschaft in Frankreich, der ADAGP, verbunden, die mit der Verwaltung der Urheberrechte von Salvador Dalí im französischen Staatsgebiet beauftragt ist.

16. Die ADAGP erhob seit 1997 die für die Verwertung des Werks von Salvador Dalí geschuldeten Beträge und leitete sie über die VEGAP an die Fundación Gala-Salvador Dalí weiter, ausgenommen jedoch die Folgerechtsvergütungen. Diese zahlte die ADAGP gemäß Art. L. 123-7 CPI, wonach die Folgerechtsvergütungen unter Ausschluss von Vermächtnisnehmern und Rechtsnachfolgern allein den Erben zustehen, unmittelbar an die Erben von Salvador Dalí. 
17. Da die Fundación Gala-Salvador Dalí und die VEGAP der Ansicht waren, dass aufgrund des Testaments von Salvador Dalí und des spanischen Rechts die aus Versteigerungen von Werken des Künstlers im französischen Staatsgebiet erzielten Folgerechtsvergütungen an sie selbst zu zahlen seien, erhoben sie am 28. Dezember 2005 vor dem Tribunal de grande instance de Paris eine entsprechende Zahlungsklage gegen die ADAGP, die ihrerseits den Erben des Malers den Streit verkündete, um die Wirkungen des zu erlassenden Urteils auf sie zu erstrecken.

18. Vor diesem Hintergrund hat das Tribunal de grande instance de Paris das Verfahren ausgesetzt und dem Gerichtshof folgende Fragen zur Vorabentscheidung vorgelegt:

1. Kann die Französische Republik nach Erlass der Richtlinie 2001/ 84 ein den Erben unter Ausschluss von Vermächtnisnehmern oder Rechtsnachfolgern vorbehaltenes Folgerecht beibehalten?

2. Gestatten die Übergangsbestimmungen des Art. 8 Abs. 2 und 3 der Richtlinie 2001/ 84 der Französischen Republik eine Ausnahmeregelung?

\section{$\mathrm{Zu}$ den Vorlagefragen}

\section{Zur Zulässigkeit des Vorabentscheidungsersuchens}

19. Die spanische Regierung und die Klägerinnen des Ausgangsverfahrens machen in ihren schriftlichen Erklärungen geltend, dass das Vorabentscheidungsersuchen unzulässig sei.

20. Zur Begründung führen sie aus, dass sich angesichts der Tatsachenlage im Ausgangsrechtsstreit die Bestimmung der Rechtsnachfolger des Urhebers des Werks im Sinne von Art. 6 Abs. 1 der Richtlinie 2001/ 84 nicht nach französischem Recht, sondern ausschließlich nach dem spanischen Erbrecht richte, da der Maler Salvador Dalí, der die spanische Staatsangehörigkeit besessen habe, an seinem Wohnsitz in Figueras in Spanien verstorben sei. Die Frage, ob Art. L. 123-7 CPI mit der Richtlinie 2001/ 84 vereinbar sei, sei darum im Ausgangsrechtsstreit, der allein dem spanischen Recht unterliege, nicht entscheidungserheblich.

21. Der Gerichtshof ist jedoch nicht befugt, im Rahmen eines Vorabentscheidungsverfahrens darüber zu entscheiden, wie nationale Vorschriften, etwa solche des internationalen Privatrechts, auszulegen sind oder ob ihre Auslegung durch das vorlegende Gericht richtig ist. Der Gerichtshof hat nämlich im Rahmen der Verteilung der Zuständigkeiten zwischen den Gemeinschaftsgerichten und den nationalen Gerichten in Bezug auf den tatsächlichen und rechtlichen Rahmen, in den sich die Vorlagefragen einfügen, von den Feststellungen in der Vorlageentscheidung auszugehen (vgl. in diesem Sinne Urteil vom 14. Februar 2008, Dynamic Medien, C-244/ 06, Slg. 2008, I-505, Randnr. 19 und die dort angeführte Rechtsprechung).
22. Dem Vorabentscheidungsersuchen ist jedoch zu entnehmen, dass es auf der Prämisse beruht, dass im Ausgangsrechtsstreit das französische Recht, insbesondere Art. L. 123-7 $\mathrm{CPI}$, anwendbar ist. Da das vorlegende Gericht Fragen nach der Auslegung von Art. 6 Abs. 1 und Art. 8 Abs. 2 und 3 der Richtlinie 2001/ 84 gestellt hat, um die Vereinbarkeit dieser Bestimmungen mit Art. L. 123-7 CPI beurteilen zu können, ist das Vorabentscheidungsersuchen für die Entscheidung über den Ausgangsrechtsstreit nicht offensichtlich unerheblich.

23. Demnach ist das Vorabentscheidungsersuchen für zulässig zu erklären.

\section{Zur Sache}

Zur ersten Frage

24. Mit seiner ersten Frage möchte das vorlegende Gericht wissen, ob Art. 6 Abs. 1 der Richtlinie 2001/ 84 dahin auszulegen ist, dass er einer innerstaatlichen Rechtsvorschrift wie Art. L. 123-7 $\mathrm{CPI}$ entgegensteht, nach der auf Folgerechtsvergütungen unter Ausschluss durch Testament eingesetzter Vermächtnisnehmer allein die gesetzlichen Erben des Künstlers Anspruch haben.

25. Einleitend ist daran zu erinnern, dass nach ständiger Rechtsprechung des Gerichtshofs bei der Auslegung einer Gemeinschaftsvorschrift nicht nur ihr Wortlaut, sondern auch ihr Zusammenhang und die Ziele zu berücksichtigen sind, die mit der Regelung, zu der sie gehört, verfolgt werden (vgl. Urteile vom 17. November 1983, Merck, 292/ 82, Slg. 1983, 3781, Randnr. 12, vom 14. Oktober 1999, Adidas, C-223/ 98, Slg. 1999, I-7081, Randnr. 23, vom 7. Juni 2005, VEMW u. a., C-17/ 03, Slg. 2005, I-4983, Randnr. 41, und vom 10. September 2009, Eschig, C-199/ 08, Slg. 2009, I-0000, Randnr. 38).

26. Es ist zunächst zu beachten, dass der Text der Richtlinie 2001/ 84 keine Erläuterung zu dem in ihrem Art. 6 Abs. 1 verwendeten Begriff der "Rechtsnachfolger" des Urhebers des Werks enthält. Mangels einer ausdrücklichen Definition dieses Begriffs ist zu prüfen, welche Ziele dem Erlass der Richtlinie 2001/ 84 zugrunde lagen.

27. Insoweit ist darauf hinzuweisen, dass für den Erlass der Richtlinie 2001/ 84 ein zweifaches Ziel maßgebend war. Zum einen sollte, wie aus den Erwägungsgründen 3 und 4 der Richtlinie hervorgeht, den Urhebern von Werken der bildenden Künste eine wirtschaftliche Beteiligung am Erfolg ihrer Werke garantiert werden, und zum anderen ging es, wie in den Erwägungsgründen 9 und 10 der Richtlinie klargestellt wird, um die Beseitigung von Wettbewerbsverzerrungen auf dem Kunstmarkt, da die Zahlung von Folgerechtsvergütungen in bestimmten Mitgliedstaaten dazu angetan war, Verkäufe von Kunstwerken in Mitgliedstaaten zu verlagern, in denen es ein Folgerecht nicht gibt. 
28. Das erste Ziel besteht darin, Künstlern ein bestimmtes Verdienstniveau zu gewährleisten. Aus diesem Grund ist nach Art. 1 Abs. 1 der Richtlinie 2001/ 84 das Folgerecht als unveräußerlich konzipiert, und es kann darauf auch im Voraus nicht verzichtet werden.

29. Die Verwirklichung dieses ersten Ziels wird aber dadurch, dass das Folgerecht nach dem Tod des Künstlers auf bestimmte Kategorien von Rechtssubjekten unter Ausschluss anderer übergeht, keineswegs beeinträchtigt, wobei dieser Übergang für dieses Ziel nur von nachgeordneter Bedeutung ist.

30. Was das zweite Ziel anbelangt, erschien es unerlässlich, im Hinblick auf Kunstwerke und Verkäufe, die vom Folgerecht erfasst werden, sowie dessen Bemessungsgrundlage und Höhe eine Harmonisierung herbeizuführen. Der Gesetzgeber wollte nämlich, wie aus dem neunten Erwägungsgrund der Richtlinie eindeutig hervorgeht, eine Situation beheben, in der sich die Verkäufe von Kunstwerken in Mitgliedstaaten konzentrierten, in denen das Folgerecht nicht oder nur in geringerer Höhe bestand als in anderen Mitgliedstaaten, wodurch die in Letzteren ansässigen Auktionshäuser oder Kunsthändler benachteiligt wurden.

31. Dieses zweite Ziel erklärt die Wahl der Rechtsgrundlage, auf der die Richtlinie 2001/ 84 erlassen wurde, nämlich Art. 95 EG. Diese Wahl bestätigt, dass sich der Erlass der Richtlinie in den Rahmen der Angleichung der Rechts- und Verwaltungsvorschriften der Mitgliedstaaten einfügt, die die Errichtung und das Funktionieren des Binnenmarkts zum Gegenstand haben. Folglich sind, wie aus den Erwägungsgründen 13 und 15 der Richtlinie 2001/ 84 hervorgeht, zwischen den nationalen Rechtsvorschriften bestehende Unterschiede, die sich nicht nachteilig auf das Funktionieren des Binnenmarkts auswirken können, nicht zu beseitigen und ist es, damit so viel Spielraum wie möglich für einzelstaatliche Entscheidungen bleibt, ausreichend, nur diejenigen nationalen Vorschriften zu harmonisieren, die sich am unmittelbarsten auf das Funktionieren des Binnenmarkts auswirken.

32. Diese Beurteilung wird durch den 27. Erwägungsgrund der Richtlinie 2001/ 84 gestützt, wonach die Intention des Unionsgesetzgebers dahin ging, dass zwar die Rechtsnachfolger des Urhebers nach dessen Tod das Folgerecht in vollem Umfang in Anspruch nehmen können, hingegen im Einklang mit dem Subsidiaritätsgrundsatz das Erbrecht der Mitgliedstaaten durch die Richtlinie unberührt bleibt, womit es jedem einzelnen Mitgliedstaat überlassen ist, die Kategorien von Personen zu bestimmen, die nach seinem nationalen Recht als Rechtsnachfolger angesehen werden können.

33. Aus dem Vorstehenden ergibt sich, dass es im Licht der mit der Richtlinie 2001/ 84 verfolgten Ziele den Mitgliedstaaten freisteht, ihre eigenen gesetzgeberischen Entscheidungen zu treffen, um die Kategorien von Personen festzulegen, denen nach dem Tod des Urhebers eines Kunstwerks das Folgerecht zugutekommen kann.
34. Darum lässt nichts in der Richtlinie 2001/ 84 den Schluss zu, dass der Unionsgesetzgeber die Anwendung von Bestimmungen ausschalten wollte, die die Koordinierung der verschiedenen innerstaatlichen Erbrechte regeln, insbesondere die der Bestimmungen des internationalen Privatrechts, die eine Rechtskollision wie die im Ausgangsrechtsstreit vorliegende lösen sollen.

35. Folglich ist es Sache des vorlegenden Gerichts, für die Anwendung der innerstaatlichen Rechtsvorschrift, durch die Art. 6 Abs. 1 der Richtlinie 2001/ 84 umgesetzt wird, ordnungsgemäß alle einschlägigen Bestimmungen zu berücksichtigen, die Kollisionen von gesetzlichen Regelungen über den für das Folgerecht geltenden Erbanfall lösen sollen.

36. Demnach ist auf die erste Frage zu antworten, dass Art. 6 Abs. 1 der Richtlinie 2001/ 84 dahin auszulegen ist, dass er nicht einer innerstaatlichen Rechtsvorschrift wie der im Ausgangsverfahren fraglichen entgegensteht, nach der auf Folgerechtsvergütungen unter Ausschluss durch Testament eingesetzter Vermächtnisnehmer allein die gesetzlichen Erben des Künstlers Anspruch haben. Es ist daher Sache des vorlegenden Gerichts, für die Anwendung der innerstaatlichen Rechtsvorschrift, durch die Art. 6 Abs. 1 der Richtlinie 2001/ 84 umgesetzt wird, ordnungsgemäß alle einschlägigen Bestimmungen zu berücksichtigen, die Kollisionen von gesetzlichen Regelungen über den für das Folgerecht geltenden Erbanfall lösen sollen.

\section{Zur zweiten Frage}

37. Mit seiner zweiten Frage möchte das vorlegende Gericht wissen, ob die Ausnahmeregelungen des Art. 8 Abs. 2 und 3 der Richtlinie 2001/ 84 dahin auszulegen sind, dass sie übergangsweise eine Beibehaltung der fraglichen Vorschrift des CPI erlauben.

38. Diese Frage ist jedoch angesichts der Antwort auf die erste Frage nicht zu beantworten.

\section{Kosten}

39. Für die Beteiligten des Ausgangsverfahrens ist das Verfahren ein Zwischenstreit in dem bei dem vorlegenden Gericht anhängigen Rechtsstreit; die Kostenentscheidung ist daher Sache dieses Gerichts. Die Auslagen anderer Beteiligter für die Abgabe von Erklärungen vor dem Gerichtshof sind nicht erstattungsfähig. 